\title{
]jfis
}

\section{Context-Aware Information Provisioning for Vessel Traffic Service Using Rule-Based and Deep Learning Techniques}

Kwang-II Kim and Keon Myung Lee

Department of Computer Science, Chungbuk National University, Cheongju, Korea

\begin{abstract}
Vessel traffic service operators (VTSOs) are primarily responsible for vessel navigation safety. However, in a few cases, it is difficult for them to focus on vessel monitoring because of the additional tasks they must perform. In recent years, decision support tools have been developed to reduce the workload of VTSOs, but vessel traffic officer requirements are not well addressed by the support tools. In this study, we survey context-aware procedures in vessel traffic service and propose an information provisioning model using rule-based and deep learning techniques. The rule-based information provisioning model uses display, prediction, and calculation objects. In case of prediction object, a deep neural network is used to predict ship near-collision and destination, and its performances are better than previous methods.
\end{abstract}

Keywords: Vessel traffic service, Decision support tool, Deep neural network, Rule-based method

\section{Introduction}

In harbor and coastal water areas, vessel traffic services (VTSs) are implemented by a VTS operator (VTSO) to improve the safety and efficiency of vessel traffic and to protect the marine environment. The tasks performed by VTSOs are primarily divided into information service (INS), navigational assistance service (NAS), and traffic organization service (TOS) [1]. These services are provided based on the experience of VTSOs.

While VTSOs monitor vessel traffic, they also frequently check the vessel entry permission, berth, and pilot schedule of inbound ships while they monitor other vessel traffic. They also support search and rescue operations (SAR) and harbor-based operations, such as cargo operation, port facilities, and navigational aids. These additional tasks interfere with their vessel monitoring tasks [2].

In recent years, in order to reduce the workload of VTSOs and the human error rate, several decision support tools have been developed to assist the decision-making abilities of VTSOs and help them to understand vessel traffic situations and how to transfer ship-based information. Praetorius and Lutzhoft [3] conducted user requirement surveys for dynamic risk management through group interviews and semi-structured interviews. Mazaheri et al. [4] proposed the detection of ship grounding candidates as a VTS decision support tool. They focused on grounding accident situations.

In this study, we survey a context-aware procedure in VTS and propose an information provisioning model using rule-based and deep learning techniques. This study enables VTSOs 
to focus on the primary task of vessel traffic management by reducing the workload associated with performing additional tasks. To display information efficiently, we developed contextbased rules to display objects based on the VTSO requirement. The objects of the proposed model include the display, prediction, and calculation of objects. These objects, with the exception of prediction, can be obtained using a rule-based approach. For prediction objects, the deep neural network (DNN) is used to predict the near-collision risk and destination of ships.

The remainder of this study is organized as follows: Section 2 analyzes the user requirements of VTSOs. In Section 3, the proposed context-aware rules and information provisioning models are described. Section 4 develops the prototype of the decision support tool and implements a performance evaluation. Finally, conclusions are presented in Section 5.

\section{User Requirement Analysis of VTS}

The International Association of Lighthouse Authorities recommendations and guidelines provide only general principles and conceptual explanations in the domain of decision support tools for VTSOs [5-7]. They address general functions and requirements; therefore, specific functions needed to support the decisions of VTSOs should be defined by a VTS authority.

According to the survey results for VTSOs performed by Kim et al. [8], VTSOs prefer to provide navigation and safety information when a ship enters a VTS area. In addition, they must assist with the analysis of information regarding collisions, ship targeting and tracking, situation awareness, and inference. VTSOs should obtain timely information from a VTS system to reduce their workload because they perform several related tasks. Moreover, this intelligent information should be automatically distributed and displayed at least 10 minutes in advance.

VTSOs are frequently required to identify the route and destination of a particular vessel and contact related offices, such as pilot, port management, and ship agents, in addition to their inherent tasks of INS, NAS, and TOS. Figure 1 shows the variation tree analysis (VTA) of a ship entry report situation, which can explain the relation between the vessel and VTSO using several information sources and decisions.

In this study, based on the user requirements obtained through VTA, the possible functions of the decision support tool are determined as follows:

- Calculation of closest point of approach (CPA)

- Calculation of estimated time of arrival (ETA)

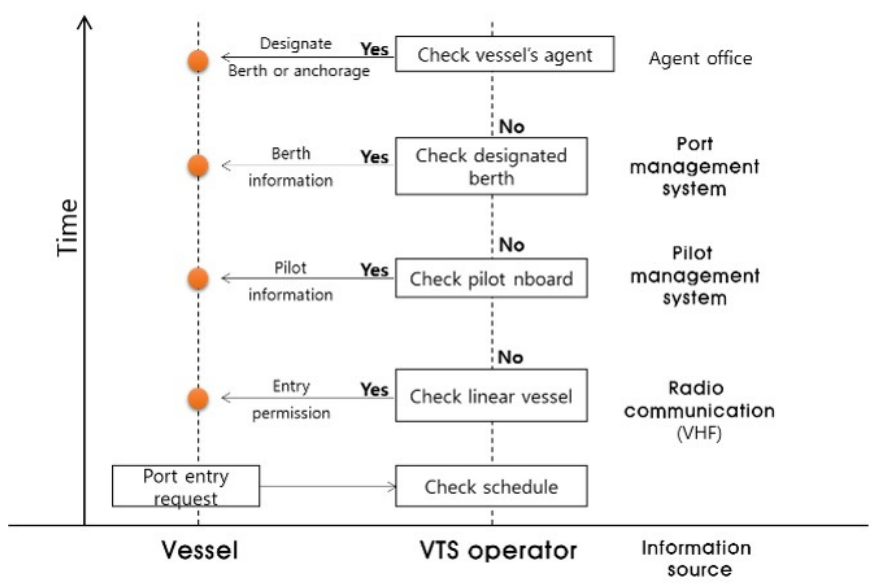

Figure 1. Variation tree analysis of entry report situation between vessel and VTSO.

- Connection to external information systems such as the Port Management Information System (PORT-MIS) and Pilot Management System (PIMS)

- Alert when a vessel navigates into dangerous areas

- Vessel historical entry/exit record

\section{Context-Aware Information Provisioning Models}

The proposed context-aware information provisioning models use rule-based and deep learning techniques. In general, a context-aware information model is a user-dependent application that discovers and reacts by changing waterway environment [9]. The rules are produced according to the contextaware information flows of VTSOs in Section 2. It allows them to rapidly and effectively obtain information using display, prediction, and calculation objects, which can enable them to concentrate on the primary task of vessel surveillance [10]. Specifically, a prediction object based on a DNN technique that trains ship trajectories and port management data can create meaningful and predictive information.

Most information in VTS is displayed partially or completely, regardless of the preference of the operator. A large amount of information can easily become confusing for VTSOs, so it easy to lose track of important events or patterns. The proposed context-aware information provisioning models can manage displayed information based on preset rules to transfer valuable information about VTS systems to the users. The rules governing the display, prediction, and calculation of objects were 


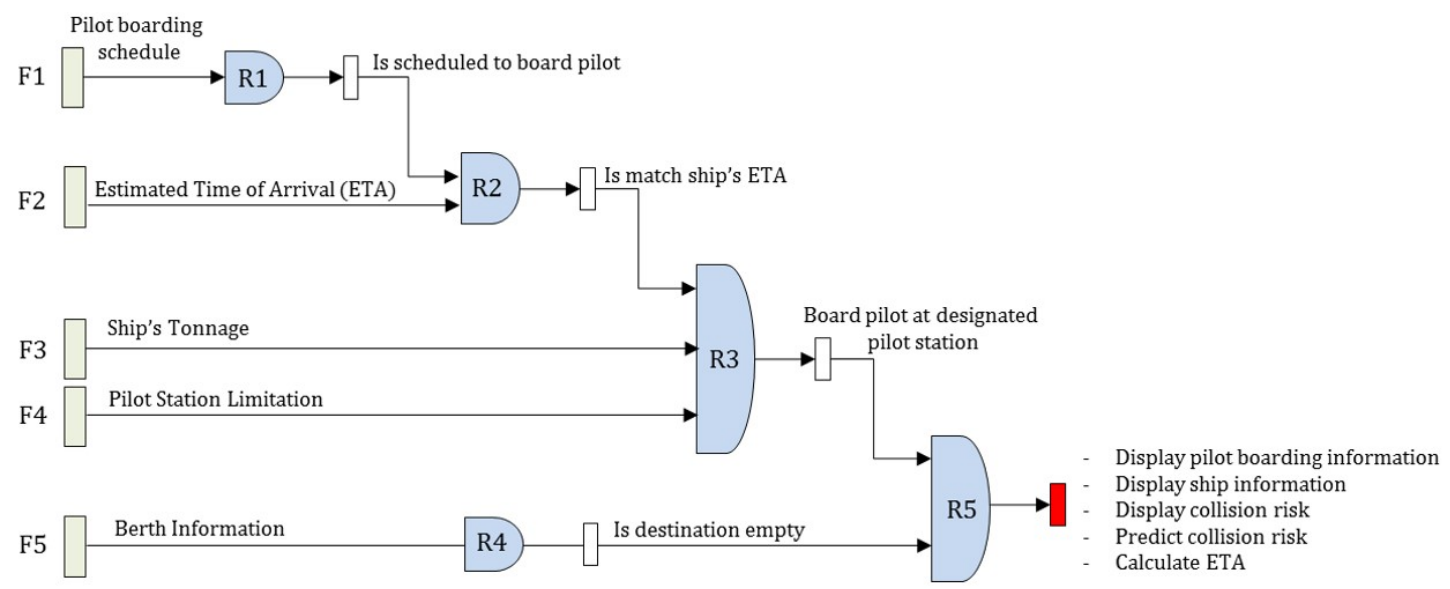

Figure 2. An example of pilot boarding information rules.

Table 1. Context-aware rules for information provisioning models

\begin{tabular}{|c|c|}
\hline & Rules: (Condition) $\rightarrow<$ Results $>$ \\
\hline 1 & $\begin{array}{l}(\text { Navigational Status }=\text { Inbound }) \wedge(\text { Entrance Report }=\text { Yes }) \wedge(\text { Entrance Permission }=\text { Yes }) \wedge(\text { Pilot } \\
\text { Schedule }=\text { Yes }) \wedge(\text { ETA report }=\text { Yes }) \wedge(\text { Pilot Schedule }- \text { ETA } \leq 30 \mathrm{~min}) \rightarrow<\text { Disp.PI, Disp.SI }, \\
\text { Disp.CR, Pred.CR, Calc.ETA }>\end{array}$ \\
\hline 2 & $\begin{array}{l}(\text { Navigational Status }=\text { Inbound }) \wedge(\text { Entrance Report }=\text { Yes }) \wedge(\text { Entrance Permission }=\text { Yes }) \wedge(\text { Pilot Schedule } \\
=\text { Yes }) \wedge(\text { ETA report }=\text { Yes }) \wedge(\text { Pilot Schedule }- \text { ETA }>30 \mathrm{~min}) \rightarrow<\text { Disp.SI, Disp.SD, Disp.CR, Pred.CR }, \\
\text { Calc.ETA }>\end{array}$ \\
\hline 3 & $\begin{array}{l}(\text { Navigational Status }=\text { Inbound }) \wedge(\text { Entrance Report }=\text { Yes }) \wedge(\text { Entrance Permission }=\text { Yes }) \wedge(\text { Pilot Schedule } \\
=\text { Yes }) \wedge(\text { Pilot exemption }=\text { Yes }) \rightarrow<\text { Disp.SI, Disp.SD, Disp.CR, Pred.CR, Calc.ETA }>\end{array}$ \\
\hline 4 & $\begin{array}{l}\text { (Navigational Status }=\text { Inbound }) \wedge(\text { Entrance Report }=\text { Yes }) \wedge(\text { Entrance Permission }=\text { Yes }) \wedge(\text { Pilot Schedule } \\
=\text { Yes }) \wedge(\text { Pilot exemption }=\text { No }) \rightarrow<\text { Disp.SI, Disp.SD, Disp.CR, Pred.CR, Calc.ETA, Call.AGNT }>\end{array}$ \\
\hline 5 & $\begin{array}{l}(\text { Navigational Status }=\text { Inbound }) \wedge(\text { Entrance Report }=\text { Yes }) \wedge(\text { Entrance Permission }=\text { No }) \rightarrow<\text { Disp.SI, } \\
\text { Disp.SD, Disp.CR, Pred.SD, Pred.CR, Calc.ETA, Call.AGNT }>\end{array}$ \\
\hline 6 & $\begin{array}{l}(\text { Navigational Status }=\text { Inbound }) \wedge(\text { Entrance Report }=\text { No }) \wedge(\text { Entrance Permission }=\text { Yes }) \rightarrow<\text { Disp.SI, } \\
\text { Disp.CR, Pred.CR, Calc.ETA }>\end{array}$ \\
\hline 7 & $\begin{array}{l}(\text { Navigational Status }=\text { Inbound }) \wedge(\text { Entrance Report }=\text { No }) \wedge(\text { Entrance Permission }=\text { No }) \rightarrow<\text { Disp.SI, } \\
\text { Disp.CR, Pred.CR, Pred.SD, Calc.ETA, Call.AGNT }>\end{array}$ \\
\hline 8 & $\begin{array}{l}(\text { Navigational Status }=\text { Outbound }) \wedge(\text { Pilot onboard }=\text { Yes }) \wedge(\text { Departure Permission }=\text { Yes }) \rightarrow<\text { Disp.PI }, \\
\text { Disp.SI, Disp.SD, Disp.CR, Pred.CR }>\end{array}$ \\
\hline 9 & $\begin{array}{l}\text { (Navigational Status }=\text { Outbound }) \wedge(\text { Pilot onboard }=\text { No }) \wedge(\text { Departure Permission }=\text { Yes }) \rightarrow<\text { Disp.PI, } \\
\text { Disp.SI, Disp.SD, Disp.CR, Pred.CR }>\end{array}$ \\
\hline 10 & $($ Navigational Status $=$ Passing $) \wedge($ Passing Report $=$ Yes $) \rightarrow<$ Disp.SI, Disp.CR, Pred.CR $>$ \\
\hline 11 & $($ Navigational Status $=$ Passing $) \bigwedge($ Passing Report $=$ Yes $) \rightarrow<$ None $>$ \\
\hline
\end{tabular}

Disp, display; Calc, calculation; Pred, prediction; SI, ship information; PI, pilot information; SD, ship destination; CR, collision risk.

created by categorizing the information into navigational status, entrance report, pilot schedule, and so on.

The display object determines whether content such as ship information, pilot information, ship destination, and collision risk display the decision support tool or not. The calculation object calculates analysis values of CPA and ETA and displays the results on the target ship [11]. The prediction object predicts the near-collision risk and ship destination candidates using a
DNN. Table 1 shows the rules used to apply the rule-based approach, which is based on VTSO user requirements.

For example, Figure 2 presents a scenario involving a boarding pilot. The information about the boarding schedule (F1), ETA (F2), tonnage (F3), pilot station (F4), and berth information (F5) is displayed if the corresponding rules (R1-R5) are satisfied. Pilot boarding information and the ETA to the pilot station are displayed when all rules are satisfied. 
Table 2. Inputs and outputs of the prediction object

\begin{tabular}{|c|c|c|}
\hline & $\begin{array}{l}\text { Estimation of ship } \\
\text { near-collision risk }\end{array}$ & $\begin{array}{c}\text { Prediction of ship } \\
\text { destination }\end{array}$ \\
\hline \multirow{7}{*}{ Input } & Course & Ship information \\
\hline & Speed & - Length \\
\hline & DCPA & - Tonnage \\
\hline & TCPA & - Nationality \\
\hline & Encounter type & - Owner \\
\hline & $\begin{array}{l}\text { Collision avoidance } \\
\text { action }\end{array}$ & $\begin{array}{l}\text { - Entrance record } \\
\text { - Next port }\end{array}$ \\
\hline & & Other berth information \\
\hline Output & $\begin{array}{c}\text { Near-collision event } \\
\text { (Categorical type) }\end{array}$ & $\begin{array}{c}\text { Ship destination code } \\
\text { (Categorical type) }\end{array}$ \\
\hline
\end{tabular}

In preparation for the prediction object and contents, several variables must be considered to predict and assess vessel movements. These variables include the ship, port, and external environment data. It is difficult to increase the accuracy in long term forecasts and recommendations by the conventional prediction models, which use the vector and hydrodynamic models of a ship.

Because VTSs store a large amount data about ship trajectories, port management, and hydrographic and metrological data over a long period of time, the proposed data driven prediction method can reflect model characteristics and expert experience. Using these data with a DNN, this study predicts the near-collision risks and the ship destination.

An input dataset is obtained from the VTS database to train the model coefficients of weights (W) and bias (B). To predict near-collision risk, it uses ship encounter variables and nearcollision event data. Ship encounter variables consist of distance to CPA (DCPA), time to CPA (TCPA), encounter type, collision avoidance action, and near-collision events [11]. Near-collision event data consist of near-collision (1) or no near-collision (0) as output variables for supervised learning. Predictions of ship destinations utilize PORT-MIS data. The target ship information and share rates of all berths are calculated when a target data ship arrives. The inputs and outputs of the DNN are represented in Table 2.

To produce meaningful predictive information based on each set of data, a multilayer perception Perceptron (MLP), which is a subset of a DNN, is used. These models consist of two parts. The first DNN model is used to predict the input of the ship's encounters data, and the second model is used to estimate the port of arrival for the ship in port. The hyper-parameter of the DNN was set with 360 hidden layers, 200 epochs, and a 0.9
Table 3. Performance of the estimation of near-collision accuracy

\begin{tabular}{ccc}
\hline & Baseline model [12] & Proposed model \\
\hline Accuracy & 0.56 & 0.72 \\
\hline
\end{tabular}

dropout probability, with the activation function of a rectified linear unit (ReLU) using the TensorFlow library in Python 3.6. Figure 3 represents process of the proposed models in VTS.

There are three databases of ship trajectory, port management, and environment variables, which are linked to the call sign and receiving time of the target ship. Input sources are preprocessed with normalization or by converting a one-hot vector. Then, the input data set is transferred to the DNN while designating the batch unit to train network model. Also, input data transfer to network model to obtain outputs.

\section{Implementation of the Proposed Models}

\subsection{Development of Decision Support Tool}

A few VTS systems are not connected to other VTS-related information systems; these include those of the harbor, cargo, and ship information, because these data are gathered by different systems. To solve this problem, the related systems for VTSs, such as PORT-MIS and PIMS, were integrated with existing ship trajectory data by setting the call sign of a ship as a foreign key in the automatic identification system (AIS) database.

We developed a decision support tool to connect the proposed DNN models. This tool can perform "display," "calculate," and "prediction" object functions according to the context-situation rules of Table 1. Near-collision risk and the prediction of the ship destination can be extracted from the proposed DNN models. Figure 4 shows the prototype of the decision support tool.

\subsection{Performance Evaluation}

To evaluate the proposed models, we compare the results of the traditional method. For the experiment of near-collision accuracy, the ship encounter data for one year pertaining to Yeosu VTS were used. The experiment of the baseline model is performed in a previous study conducted by Kim et al. [12]. That experimental environment applied logistic regression with the same sample data. The accuracy is calculated by comparing the predicted output with real data. Table 3 indicates the performance of the near-collision accuracy. The proposed model of near-collision accuracy is $16 \%$ higher than the baseline model.

For prediction accuracy of the ship destination, the ship entry and departure logs of the PORT-MIS database from 1999-2015 


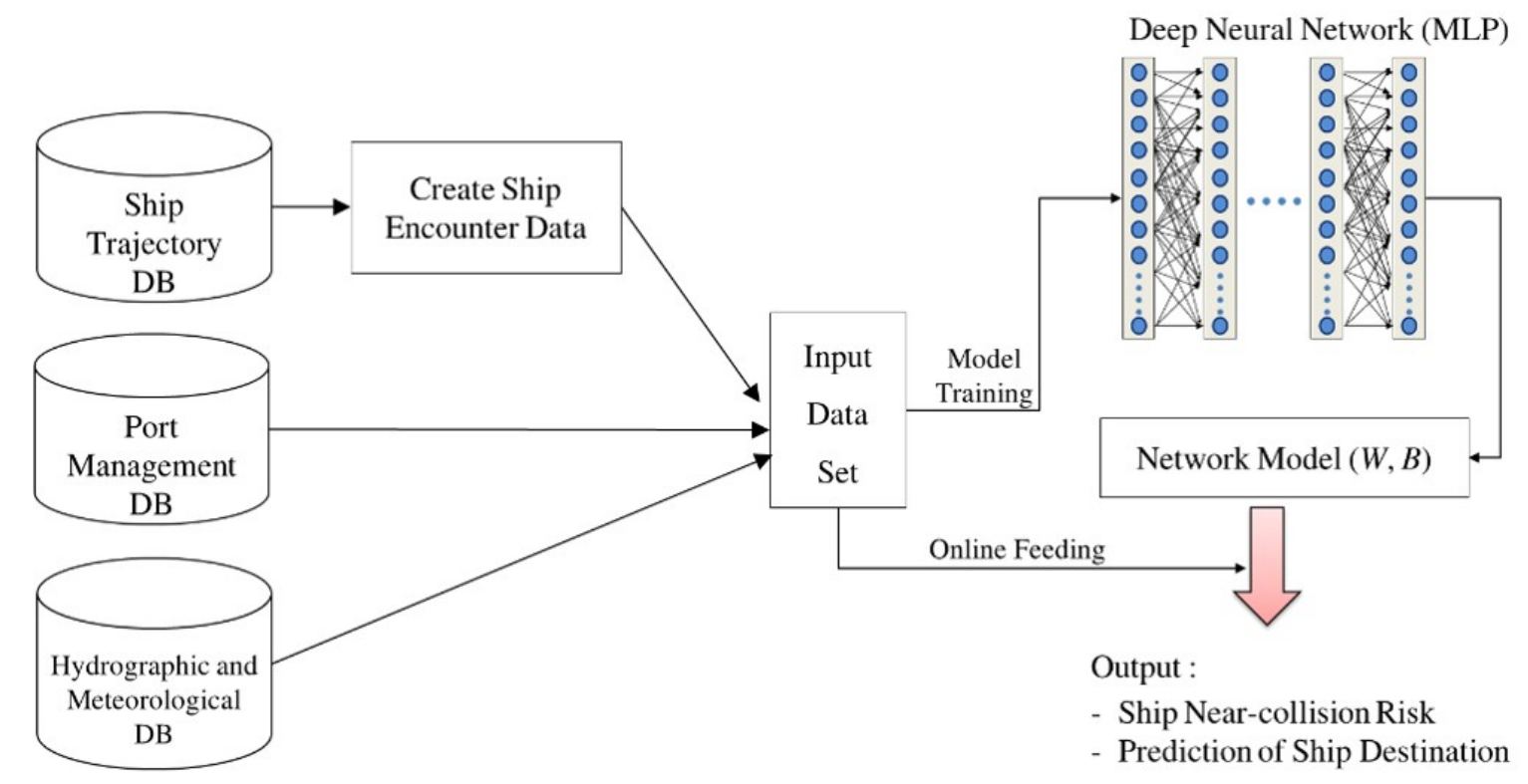

Figure 3. Deep neural network models for near-collision risk evaluation and ship destination prediction.

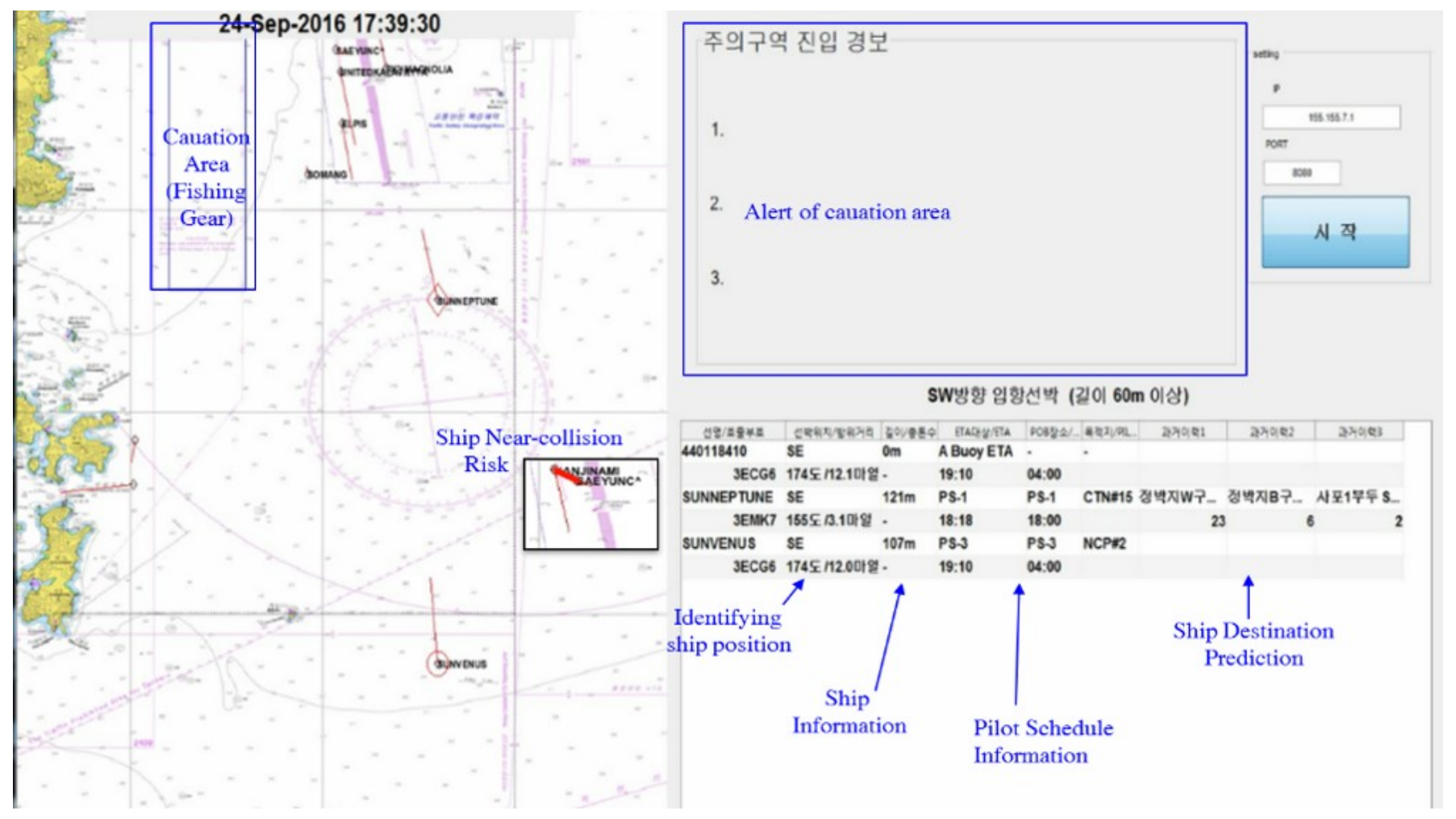

Figure 4. Prototype of decision support tool.

were used. Each transaction has a ship destination for which the number of berths and anchorages is 353 . The results obtained from the experiment with the proposed DNN model show that the accuracy is $58 \%$ in the top 1 selection and $76 \%$ within top 3 selections. The comparison experiments performed to evaluate model performance were prepared using the top 1 frequent and within top 3 frequents instead of the DNN results. The proposed model for the prediction of ship destination accuracy is $21 \%$ higher in the top 1 and $24 \%$ higher in top 3 than baseline model (Figure 4). 
Table 4. Performance of the prediction of ship destination

\begin{tabular}{ccc}
\hline & Baseline model & Proposed model \\
\hline Top 1 accuracy & 0.37 & 0.58 \\
Top 3 accuracy & 0.52 & 0.76 \\
\hline
\end{tabular}

\section{Conclusions}

This study has proposed a context-aware information provisioning model for VTS by applying rule-based and deep learning techniques. The overall goal is to effectively display abstract information and to integrate related systems with a VTS system by connecting ship traffic databases under single window monitoring. In particular, maritime traffic situations are more congested and vessel traffic routes change based on season, economy, and government policy. Therefore, the proposed models that use context-aware rules and DNN techniques can reflect diverse vessel traffic situations.

\section{Conflict of Interest}

No potential conflict of interest relevant to this article was reported.

\section{Acknowledgements}

This research was supported by Next-Generation Information Computing Development Program through the National Research Foundation of Korea (No.: NRF-2017M3C4A7069 432) and by Basic Science Research Programs through the National Research Foundation of Korea funded by the Ministry of Education (No. NRF-2016R1A6A3A11935806 and NRF-2015R1D1 A1A01061062).

\section{References}

[1] International Maritime Organization, Resolution A.857(20): Guidelines for Vessel Traffic Services, 1997.

[2] J. S. Jeong, G. K. Park, and J. S. Kim, "Prediction table to improve maritime situation awareness by VTS operator," in Proceedings of International Conference on Fuzzy Theory and Its Applications, 2013, pp. 479-481. https://doi.org/10.1109/iFuzzy.2013.6825487

[3] G. Praetorius and M. Lutzhoft, "Decision support for vessel traffic service (VTS): user needs for dynamic risk management in the VTS," Work, vol. 41(Supplement 1), pp. 4866-4872, 2012. https://doi.org/10.3233/WOR-20120779-4866

[4] A. Mazaheri, F. Goerlandt, J. Montewka, and P. Kujala, “A decision support tool for VTS centers to detect grounding candidates," International Journal on Marine Navigation and Safety of Sea Transportation, vol. 6, no. 3, pp. 337343, 2012.

[5] International Association of Lighthouse Authorities, Use of Decision Support Tools for VTS Personnel (No. 1110), 2014.

[6] International Association of Lighthouse Authorities, Preparation of Operational and Technical Performance Requirements for VTS Systems (No. 1111), 2015.

[7] International Association of Lighthouse Authorities, Operational and Technical Performance of VTS Systems (Recommendation V-128), 2016.

[8] H. Kim, S. Kim, N. Sohn, J. Oh, and M. Lee, "Survey analysis for user requirement analysis in decision support system," in Proceedings of the Spring Conference for Korean Institute of Navigation and Port Research, Busan, Korea, 2011, pp. 109-113.

[9] K. M. Lee, B. K. Sohn, J. T. Kim, S. W. Lee, J. H. Lee, J. W. Jeon, and J. Cho, "An SoC-based context-aware system architecture," in Knowledge-Based and Intelligent Information and Engineering Systems. Heidelberg: Springer, 2004, pp. 573-580. https://doi.org/10.1007/9783-540-30134-9_77

[10] K. I. Kim and K. M. Lee, "Ship encounter risk evaluation for coastal areas with holistic maritime traffic data analysis," Advanced Science Letters, vol. 23, no. 10, pp. 9565-9569, 2017. https://doi.org/10.1166/asl.2017.9748

[11] K. M. Lee and K. M. Lee, "Agent-based knowledge evolution management and fuzzy rule-based evolution detection in Bayesian networks," in Proceedings of the International Conference on Fuzzy Theory and Its Applications, Taipei, Taiwan, 2013, pp. 146-149. https: //doi.org/10.1109/iFuzzy.2013.6825426

[12] K. I. Kim, J. S. Jeong, and B. G. Lee, "Study on the analysis of near-miss ship collisions using logistic regression,' Journal of Advanced Computational Intelligence and Intelligent Informatics, vol. 21, no. 3, pp. 467-473, 2017. https://doi.org/10.20965/jaciii.2017.p0467 


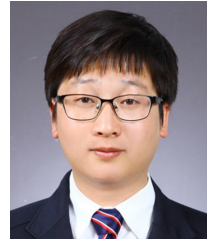

Kwang-Il Kim received his B.S., M.S., and Ph.D. in Maritime Information System Engineering from Mokpo National Maritime University, Mokpo Korea, in 2005, 2010, and 2012, respectively. Now he works at Research Institute for Computer and Information Communication of Chungbuk National University as a visiting professor.

E-mail: kikim82@cbnu.ac.kr

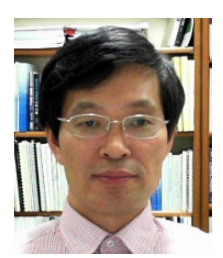

Keon Myung Lee is a professor in the Department of Computer Science, Chungbuk National University, Korea. He received his B.S., M.S., and Ph.D. degrees in computer science from KAIST, Korea and was a postdoctorate fellow at INSA de Lyon, France. He was a visiting professor at the University of Colorado at Denver and a visiting scholar at Indiana University, USA. His principal research interests are data mining, machine learning, soft computing, big data processing, and intelligent service systems.

E-mail: kmlee@cbnu.ac.kr 